\title{
ON THE ABSOLUTES OF COMPACT SPACES WITH A MINIMALLY ACTING GROUP
}

\author{
INGO BANDLOW
}

(Communicated by Franklin D. Tall)

\begin{abstract}
If an $\omega$-bounded group $G$ acts continuously on a compact Hausdorff space $X$ and the orbit of every point is dense in $X$, then $X$ is coabsolute to a Cantor cube.
\end{abstract}

A topological group $G$ acts continuously on the topological space $X$ if $G$ is a group of homeomorphisms of $X$ and the natural map $X \times G \rightarrow X$ is continuous. $X$ is always assumed to be a compact Hausdorff space. We say that $G$ acts minimally on $X$ if the orbit $\{g(x): g \in G\}$ is dense in $X$ for every point $x \in X$. It is easy to see that $G$ acts minimally on $X$ if for every nonempty open subset $U$ of $X$, there exist $g_{1}, \ldots, g_{n} \in G$ such that $g_{1}(U) \cup \cdots \cup g_{n}(U)=X$.

Balcar and Blaszczyk proved in [2] that every zero-dimensional compact space with a minimally acting countable group is coabsolute to a Cantor cube. Two compact spaces are said to be coabsolute if their respective Boolean algebras of regular open subsets are isomorphic. From results of Uspenskij [14] and Shapiro [12] it follows that if an $\omega$-bounded group $G$ acts transitively and continuously on a compact space $X$, then $X$ is coabsolute to a Cantor cube. A topological group $G$ is said to be $\omega$-bounded if and only if for any neighbourhood $U$ of its neutral element there is a countable subset $A$ of $G$ such that $G=A U$ (Guran, see Archangelskij [1]).

The aim of this note is to prove the following

Theorem 1. If an $\omega$-bounded group $G$ acts continuously and minimally on the compact Hausdorff space $X$, then $X$ is coabsolute to a Cantor cube.

The proof is based on results of Shapiro $[12,13]$ and makes use of arguments of Uspenskij [14] and Balcar and Blaszczyk [2].

At first, we need the following outstanding result of Shapiro:

(1) Every dyadic compact space which is homogeneous with respect to the weight is coabsolute to a Cantor cube.

A space $X$ is said to be homogeneous with respect to the weight if $w(U)=$ $w(X)$ for every nonempty open subset $U$.

Received by the editors June 23, 1992.

1991 Mathematics Subject Classification. Primary 54D80, 22A05.

Key words and phrases. Coabsolute spaces, Dugundji space, $\omega$-bounded group.

(C) 1994 American Mathematical Society $0002-9939 / 94 \$ 1.00+\$ .25$ per page 
An interesting subclass of dyadic compacta is the class of Dugundji spaces introduced by Pelczynski [10]. We refer to Haydon [8] or Scepin [11] for basic material on Dugundji spaces. Shapiro [13] proved the following fact:

(2) A compact Hausdorff space $X$ is coabsolute to a Dugundji space iff $X$ is the inverse limit of a continuous inverse system $\left\langle X_{\alpha}, \pi_{\alpha}^{\beta}, \rho\right\rangle$ where $\left|X_{0}\right|=1$, each $\pi_{\alpha}^{\alpha+1}$ has weight $\leq \omega$, and each $\pi_{\alpha}^{\beta}$ is skeletal.

The weight of a continuous map $f: X \rightarrow Y$ is defined to be the minimal cardinality of a system $\gamma$ of open cozero subsets of $X$ such that $\gamma \cup\left\{f^{-1} U: U\right.$ is open in $Y$ \} is a subbase for the topology in $X$ (Pasynkov [9]). A map $f: X \rightarrow$ $Y$ is skeletal if for every nonempty open subset $U$ of $X$ the set $\operatorname{Int}(\operatorname{cl}(f U))$ is nonempty. Every regular closed subset of a dyadic space is dyadic too (Efimov [7]). Further, the weight and the $\pi$-weight of dyadic spaces coincide. An easy consequence of $(1)$ is:

(3) Every compact space $X$ which is coabsolute to a Dugundji space and is homogeneous with respect to the $\pi$-weight is coabsolute to a Cantor cube.

It is obvious, that if a group acts minimally on a space $X$, then $X$ is homogeneous with respect to the $\pi$-weight.

To prove that a compact space satisfying the conditions of Theorem 1 is coabsolute to a Dugundji space, we make use of elementary substructures. For a good introduction to elementary substructures see Baumgartner [5] or Dow [6].

There is always a closed unbounded family of "suitable" countable elementary substructures of $\mathscr{H}_{\theta}$, where $\theta$ is a sufficiently large regular cardinal. "Suitable" means that all necessary "information" can be found in the elementary substructure. The following fact is well known and very important for applications of elementary substructures:

(4) If $\mathscr{M}$ is an elementary substructure of $\mathscr{H}_{\theta}$ and $A \in \mathscr{M}$ is a countable set, then $A \subset \mathscr{M}$.

A discussion of the construction we are now going to describe can be found in Bandlow [3].

Let $X$ be a compact Hausdorff space, $\theta$ a sufficiently large regular uncountable cardinal, and $\mathscr{M}$ a suitable elementary substructure of $\mathscr{K}_{\theta}$. Let $I$ denote the unit segment $[0,1] . \phi_{\mathscr{K}}^{X}$ denotes the interior product of all continuous functions $f: X \rightarrow I$ which are elements of $\mathscr{M}$, i.e.,

$$
\phi_{\mathscr{M}}^{X}=\otimes(C(X, I) \cap \mathscr{M}) .
$$

Set $X(\mathscr{M})=\phi_{\mathscr{M}}^{X}(X)$. Remark, that $\phi_{\mathscr{M}}^{X}\left(x_{1}\right) \neq \phi_{\mathscr{M}}^{X}\left(x_{2}\right)$ iff $f\left(x_{1}\right) \neq f\left(x_{2}\right)$ for a function $f \in \mathscr{C}(X, I) \cap \mathscr{M}$.

Proposition 2. A compact Hausdorff space $X$ is coabsolute to a Dugundji space iff there is a closed unbounded family $D$ of countable elementary substructures of $\mathscr{H}_{\theta}$, where $\theta$ is a sufficiently large regular uncountable cardinal, such that for every set $T \subseteq D$ the interior product $\otimes\left\{\phi_{\mathscr{M}}^{X}: \mathscr{M} \in T\right\}$ is a skeletal map.

For our purpose we need only one direction of this assertion. For this reason we refer the reader to Bandlow [4] for the complete proof.

Proof (sufficiency). Fix a transfinite sequence $\mathscr{M}_{\alpha} \in D, \alpha<\rho$, such that for every pair of distinct points $x, y \in X$ there is an $\alpha<\rho$ and a continuous 
function $f: X \rightarrow I, f \in \mathscr{M}_{\alpha}$, with $f(x) \neq f(y)$. Set $\pi^{\alpha}=\otimes\left\{\phi_{\mathscr{M}_{\gamma}}^{X}: \gamma<\alpha\right\}$ and $X_{\alpha}=\pi^{\alpha}(X)$.

If $\alpha<\beta$, there is a map $\pi_{\alpha}^{\beta}: X_{\beta} \rightarrow X_{\alpha}$. Obviously, $X$ is the limit space of the system $\left\langle X_{\alpha}, \pi_{\alpha}^{\beta}, \rho\right\rangle$. By the definition of $\pi^{\alpha}$, if $V \in \mathscr{M}_{\gamma}, \gamma<\alpha$, is a cozero subset of $X$, i.e., there is an $f \in C(X, I) \cap \mathscr{M}_{\gamma}$ with $f^{-1}(0,1]=V$, then $\left(\pi^{\alpha}\right)^{-1} \pi^{\alpha}(V)=V$ and $\pi^{\alpha}(V)$ is a cozero subset of $X_{\alpha}$. Set

$$
\gamma_{\alpha}=\left\{\pi^{\alpha+1}(V): V \in \mathscr{M}_{\alpha} \backslash \bigcup\left\{\mathscr{M}_{\gamma}: \gamma<\alpha\right\} \text { and } V \text { is a cozero subset of } X\right\} .
$$

Then $\gamma_{\alpha} \cup\left\{\left(\pi_{\alpha}^{\alpha+1}\right)^{-1} U: U\right.$ is an open subset of $\left.X_{\alpha}\right\}$ is a subbase of $X_{\alpha+1}$. Hence, $w\left(\pi_{\alpha}^{\alpha+1}\right) \leq \omega$.

Since $\pi^{\alpha}$ is skeletal, $\pi_{\alpha}^{\beta}$ is skeletal too for every $\beta$ with $\alpha<\beta<\rho$.

Lemma 3. If an $\omega$-bounded group $G$ acts continuously on the compact Hausdorff space $X$, then for every $g \in G$ and every suitable elementary substructure $\mathscr{M}$ there is an homeomorphism $g_{\mathscr{K}}: X(\mathscr{M}) \rightarrow X(\mathscr{M})$ such that $g_{\mathscr{M}} \circ \phi_{\mathscr{M}}^{X}=\phi_{\mathscr{M}}^{X} \circ g$. Proof. Let $x, y$ be arbitrary points of $X$ with $\phi_{\mathscr{M}}^{X}(x)=\phi_{\mathscr{M}}^{X}(y)$. We have to prove that

$$
\phi_{\mathscr{M}}^{X}(g(x))=\phi_{\mathscr{M}}^{X}(g(y)) \text { for every } g \in G .
$$

For this reason we check that $f(g(x))=f(g(y))$ for every $f \in C(X) \cap \mathscr{M}$.

Consider the map $f^{*}: G \rightarrow C(X)$ where $f^{*}(g)=f \circ g$ for each $g \in G$. Here we treat $C(X)$ as a Banach space with the usual norm. Then $f^{*}$ is continuous. We prove that $f^{*}(G)$ is separable.

For each $n \in \mathbb{N}$ let $U_{n}$ be a symmetric neighbourhood of the neutral element in $G$ such that $\|f \circ h-f\|<1 / n$ for each $h \in U_{n}$. Fix countable sets $A_{n} \subseteq G$ with $A_{n} U_{n}=G, n=1,2,3, \ldots$ Remark, that $U_{n} B_{n}=G$, where $B_{n}=A_{n}^{-1}$. For every $h \in U_{n}$ and $b \in B_{n}$ we have $\|f \circ(h \circ b)-f \circ b\|<1 / n$. Hence, $K=\left\{f \circ b: b \in \bigcup B_{n}\right\}$ is a dense subset of $f^{*}(G)$. Since $f \in \mathscr{M}$, we may assume that $K \in \mathscr{M}$ and, by assertion (4), $K \subseteq C(X) \cap \mathscr{M}$. Consequently, for every $g \in G$ and every $\varepsilon>0$ there is a function $h \in C(X) \cap \mathscr{M}$ such that $\|f \circ g-h\|<\varepsilon$. From $\phi_{\mathscr{M}}^{X}(x)=\phi_{\mathscr{M}}^{X}(y)$ it follows that $h(x)=h(y)$ for every $h \in C(X) \cap \mathscr{M}$. Hence, $f(g(x))=f(g(y))$.

This proves that for every $g \in G$ there is a map $g_{\mathscr{K}}: X(\mathscr{M}) \rightarrow X(\mathscr{M})$ such that $g_{\mathscr{M}} \circ \phi_{\mathscr{M}}^{X}=\phi_{\mathscr{K}}^{X} \circ g$. Since all spaces are compact, $g_{\mathscr{M}}$ is continuous. It is easy to see that $g_{\mathscr{M}} \circ h_{\mathscr{M}}=(g \circ h)_{\mathscr{M}}$ for arbitrary $g, h \in G$. Hence, $\left\{g_{\mathscr{K}}: g \in G\right\}$ is a group of homeomorphisms of $X(\mathscr{M})$.

Proof of Theorem 1. Let $\theta$ be a sufficiently large regular uncountable cardinal, $D$ a closed unbounded family of suitable countable elementary substructures of $\mathscr{H}_{\theta}$, and $T$ a subset of $D$. By Proposition 2, we have to prove that $\varphi=$ $\otimes\left\{\phi_{\mathscr{M}}^{X}: \mathscr{M} \in T\right\}$ is skeletal.

Set $X^{\prime}=\varphi(X)$. Applying Lemma 3, find an homeomorphism $g^{\prime}: X^{\prime} \rightarrow X^{\prime}$ such that $g^{\prime} \circ \varphi=\varphi \circ g$. Assume, on the contrary, that there is a nonempty open subset $U$ of $X$ with Int $\varphi(U)=\varnothing$. Since $G$ acts minimally on $X$, there are $g_{1}, \ldots, g_{n} \in G$ such that $g_{1}(U) \cup \cdots \cup g_{n}(U)=X$. Set $\gamma=\left\{V: \varnothing \neq V \subseteq X^{\prime}\right.$ is open and $V \cap \varphi(U)=\varnothing\}$.

Then $U \gamma$ is a dense subset of $X^{\prime}$. There exist $V_{1}, \ldots, V_{n} \in \gamma$ such that 


$$
\begin{aligned}
g_{1}^{\prime}\left(V_{1}\right) \cap \cdots & \cap g_{n}^{\prime}\left(V_{n}\right) \neq \varnothing . \text { Then } \\
\varnothing & =\left(g_{1}^{\prime}(\varphi(U)) \cap g_{1}^{\prime}\left(V_{1}\right)\right) \cup \cdots \cup\left(g_{n}^{\prime}(\varphi(U)) \cap g_{n}^{\prime}\left(V_{n}\right)\right) \\
& \supseteq\left(g_{1}^{\prime}(\varphi(U)) \cup \cdots \cup g_{n}^{\prime}(\varphi(U))\right) \cap\left(g_{1}^{\prime}\left(V_{1}\right) \cap \cdots \cap g_{n}^{\prime}\left(V_{n}\right)\right) \\
& \supseteq\left(\varphi\left(g_{1}(U)\right) \cup \cdots \cup \varphi\left(g_{n}(U)\right)\right) \cap\left(g_{1}^{\prime}\left(V_{1}\right) \cap \cdots \cap g_{n}^{\prime}\left(V_{n}\right)\right) \neq \varnothing .
\end{aligned}
$$

This is the desired contradiction.

\section{REFERENCES}

1. A. V. Archangelskij, Classes of topological groups, Russian Math. Surveys 36 (1981), 151-174.

2. B. Balcar and A. Blaszczyk, On minimal dynamical systems on Boolean algebras, Comment. Math. Univ. Carolinae 31 (1990), 7-11.

3. I. Bandlow, A construction in set theoretic topology by means of elementary substructures, Z. Math. Logik Grundlag. Math. 37 (1991).

4. -Math. Phys. 31 (1992).

5. J. Baumgartner, Applications of the proper forcing axiom, Handbook of Set-Theoretic Topology, North-Holland, Amsterdam, 1984, pp. 913-960.

6. A. Dow, An introduction to applications of elementary submodels to topology, Topology Proc. 13 (1988).

7. B. A. Efimov, Dyadic bicompacta, Trudy Moscov. Mat. Obshch. 14 (1965), 211-247.

8. R. Haydon, On a problem of Pelczynski: Miljutin spaces, Dugundji spaces and AE(0-dim), Studia Math. 52 (1976), 23-31.

9. B. A. Pasynkov, Dokl. Akad. Nauk SSSR 221 (1975), 543-546.

10. A. Pelczynski, Linear extensions, linear averagings and their applications to linear topological classifications of spaces of continuous functions, Dissertationes Math. 58 (1968).

11. E. V. Scepin, Topology of limit spaces of uncountable inverse spectra, Uspekhi Mat. Nauk 31 (1976), no. 5, 191-226.

12. L. B. Shapiro, On absolutes of topological spaces and continuous mappings, Dokl. Akad. Nauk SSSR 226 (1976), no. 3, 523-526.

13. __ On spaces coabsolute to dyadic bicompacta, Dokl. Akad. Nauk SSSR 293 (1987), no. 5, 1077-1081.

14. V. V. Uspenskij, Why compact groups are dyadic, General Topology and its Relations to Modern Analysis and Algebra, Proc. Sixth 1986 Prague Topological Symposium, Heldermann Verlag, Berlin, 1988, pp. 601-610.

ERnst-Moritz-ARndt Universität Greifswald, Fachbereich Mathematik, 17489 GreifSWALD, JAHNSTRASSE 15A, GERMANY 